\title{
100-GHZ BAND TEST OBSERVATIONS OF THE KVN 21-M RADIO TELESCOPES
}

Kee-Tae Kim, Do-Young Byun, Do-Heung Je, Seog-Oh Wi, Jae-Han Bae, Tae-Hyun Jung, Chang-Hoon Lee, Seog-Tae Han, Min-Gyu Song, Jae-hoon Jung, Hyun-Soo Chung, Hyo-Ryung Kim, and Bong Gyu Kim Korea Astronomy and Space Science Institute, Daedeokdaero 776, Yuseong, Daejeon 305-348, Korea E-mail : ktkim@kasi.re.kr

(Received December 02, 2010; Revised March 16, 2011; Accepted April 14, 2011)

\begin{abstract}
We carry out 100-GHz band test observations with the newly-constructed KVN 21-m radio telescopes in order to evaluate their performance. The three telescopes have similar performance parameters. The pointing accuracies are about $4^{\prime \prime} \mathrm{rms}$ for the entire sky. The main beam sizes are about $30^{\prime \prime}$ (FWHMs), which is nearly the diffraction limit of the telescopes at the observing frequency $(97 \mathrm{GHz})$. The measured aperture and main-beam efficiencies are about $52 \%$ and $46 \%$, respectively, for all three telescopes. The estimated moon efficiency is $\sim 84 \%$ for the KVN Tamna telescope. The first sidelobes appear 50" $(\sim 1.6 \times \mathrm{FWHM})$ from the main beam centers and the levels are on average $-14 \mathrm{~dB}$.
\end{abstract}

Key words : instruments: radio telescope

\section{INTRODUCTION}

The Korean VLBI Network (KVN) consists of three 21-m radio telescopes, which are located in Seoul (Yonsei University), Ulsan (University of Ulsan), and Jeju island (Tamna University) (Kim et al. 2004). The three baselines are 305,359 , and $478 \mathrm{~km}$. KVN is a compact VLBI network, compared to the Very Long Baseline Array (VLBA) and the European VLBI Network $(\mathrm{EVN})$, both of which have the longest baselines $>$ $5000 \mathrm{~km}$. In order to obtain more and larger baselines, $\mathrm{KVN}$ will be often run in combination with Japanese and Chinese VLBI networks at 22 and $43 \mathrm{GHz}$. The socalled East Asian VLBI Network (EAVN) is expected to be comparable to VLBA or EVN in spatial resolution, sensitivity, and imaging capability (e.g., Yi \& Jung 2008). In addition, KVN will be operated in four $(22,43,86,129 \mathrm{GHz})$ different frequency bands simultaneously using multi-frequency band receiver systems (Han et al. 2008). Simultaneous multi-frequency observations will make it possible to use the $22 \mathrm{GHz}$ data for performing phase calibrations of higher-frequency data (Jung et al. 2011; see also Asaki et al. 1998; Middelberg et al. 2005). By this multi-frequency phase referencing technique, KVN could play an important role in millimeter VLBI observations of maser sources in galactic star-forming regions and evolved stars and active galactic nuclei.

The KVN antennae were built by Antedo Inc. in collaboration with its domestic counterpart, High Gain Antenna, between 2006 September and 2008 December (Fig. 1). In this paper we present the results of 100$\mathrm{GHz}$ band test observations undertaken with the KVN

Corresponding Author: K.-T. Kim
Table 1.

Specification of KVN antennae

\begin{tabular}{ll}
\hline \hline Parameter & Specification \\
\hline Main Reflector & $21.03 \mathrm{~m}$ \\
Subreflector & $2.25 \mathrm{~m}$ \\
Maximum Speed & $3 \mathrm{~m} \mathrm{sec}^{-1}$ \\
Maximum Acceleration & $3 \mathrm{~m} \mathrm{sec}^{-2}$ \\
Fast Switching & $<13 / 26 \mathrm{sec}$ \\
Operating Ranging & $\mathrm{AZ:} \pm 270^{\circ}, \mathrm{EL}: 0^{\circ}-90^{\circ}$ \\
\hline \multicolumn{2}{c}{ a $<13$ sec for a $10^{\circ}$ switching at EL $\leq 60^{\circ}$ while $<26 \mathrm{sec}$ at } \\
$60^{\circ}<\mathrm{EL} \leq 80^{\circ}$.
\end{tabular}

21-m radio telescopes. For the acceptance test of the completed KVN antennae, we measured the pointing accuracies and various other efficiencies. This paper is organized as follows. Sections 2 and 3 describe the details of the telescope system, and the telescope and instrument software. Section 4 presents the results of the pointing accuracy measurements, while Section 5 gives the results of the efficiency measurements. The last section summarizes the main results.

\section{TELESCOPE SYSTEM}

\section{$2.1 \quad$ Antennae}

KVN radio telescopes are shaped Cassegrain antennae with $21.03 \mathrm{~m}$ main reflectors and $2.25 \mathrm{~m}$ subreflec- 


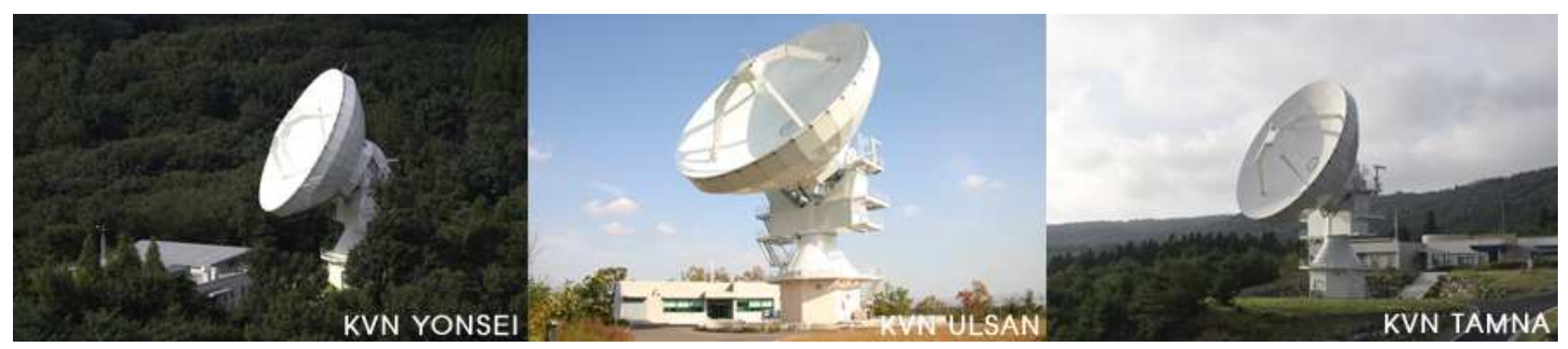

Fig. 1. - KVN radio observatories with the 21-m telescopes. (Left) KVN Yonsei radio observatory, (Middle) KVN Ulsan radio observatory, (Right) KVN Tamna radio observatory.

tors. The $\mathrm{f} / \mathrm{D}$ of the antenna optics is 4.0. The main and sub- reflectors are slightly modified from the usual parabolic and hyperbolic surfaces, respectively, in such a way that the illumination of the main reflector by the receiver feed horn is uniform rather than tapered at the edge as for the conventional Cassegrain ones. Consequently, they have higher aperture efficiencies and smaller full-width at half maximums (FWHMs) at the cost of sidelobe levels than Cassegrain antennae with main- and sub-reflectors of the same size (see Section 5). Table 1 delineates the specification of the telescopes. More detailed information of the geometry and dimensions can be found in Chung et al. (2006).

The subreflector was molded from aluminum as a single piece with a surface accuracy of $50 \mu \mathrm{m}$. The main reflector comprises of 200 aluminum panels. The surface accuracies of the individual panels are $\sim 65 \mu \mathrm{m}$. The panels were installed on the iron backup structure by four adjusters in 6 circumferential rows and then were aligned to match a theoretically designed surface by photogrammetry at $48^{\circ}$ elevation. The alignment errors were 50,54 , and $51 \mu \mathrm{m}$ for the KVN Yonsei, Ulsan, Tamna antennae, respectively. The total surface accuracies of the main reflectors are expected to be $\sim 120 \mu \mathrm{m}$ at the adjusted elevation under the condition that deformations due to gravity and thermal effects are compensated with the subreflector adjustment. The alignment error was measured at $0^{\circ}$ elevation as well, in order to determine deformations of the main reflectors due to gravity. The value was about $120 \mu \mathrm{m}$. In that case the total accuracy is roughly $160 \mu \mathrm{m}$. The details of the constructions of KVN antennae will be presented in a separate paper (Wi et al., in preparation)

\subsection{Antenna Optics and Receiver}

The signal from the subreflector arrives at the receiver feed horn, which is installed at the effective shaped Cassegrain focus, through a vertex window and a $45^{\circ}$ mirror. The vertex window is made of styrofoam and was checked to be free from appreciable loss, $<1 \%$. The corrugated horn was designed and fabricated to have $17 \mathrm{~dB}$ edge taper for reducing the spillover past the subreflector (Green 1963). The signal is amplified

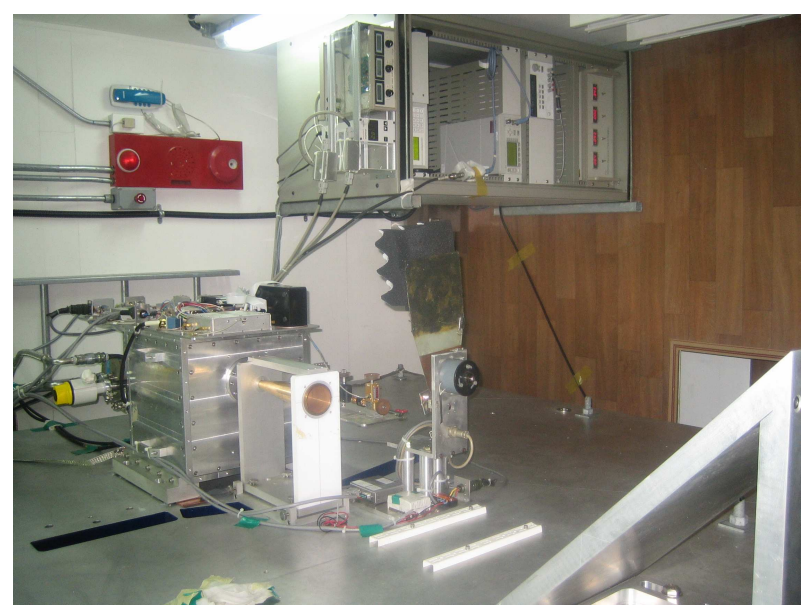

Fig. 2.- Inside the receiver room of the KVN Ulsan radio telescope, which is pointed to the zenith. The 100$\mathrm{GHz}$ band receiver is seen with the feed horn on the receiver plate, while the $45^{\circ}$ mirror is on the right bottom corner. The vertex window is above the mirror.

by cryogenic HEMT amplifiers on $15 \mathrm{~K}$ stage of the dewar. The receiver operates from $84 \mathrm{GHz}$ to $110 \mathrm{GHz}$ using a room-temperature biasable mixer. This DSB (Double SideBand) mixer converts the amplified signal to $1.4 \mathrm{GHz}$ IF (Intermediate Frequency) band with 600 $\mathrm{MHz}$ bandwidth.

The feed horn was carefully aligned using a theodolite at the vertex window and a small optical mirror at the surface of $45^{\circ}$ mirror. For simple optics we used a big feed horn at the room-temperature stage instead of a small feed horn at the cold stage together with additional optical components. As a result, alignment of the horn was straightforward, but the loss of the horn significantly increased the receiver noise temperature. The measured receiver temperatures are typically 310 $\mathrm{K}$ and $220 \mathrm{~K}$ at $87.64 \mathrm{GHz}$ and $97 \mathrm{GHz}$ local oscillator frequency, respectively.

\subsection{Backends}

A filterbank was used for the measurement of pointing accuracy by observing $\mathrm{SiO}$ maser sources in the 

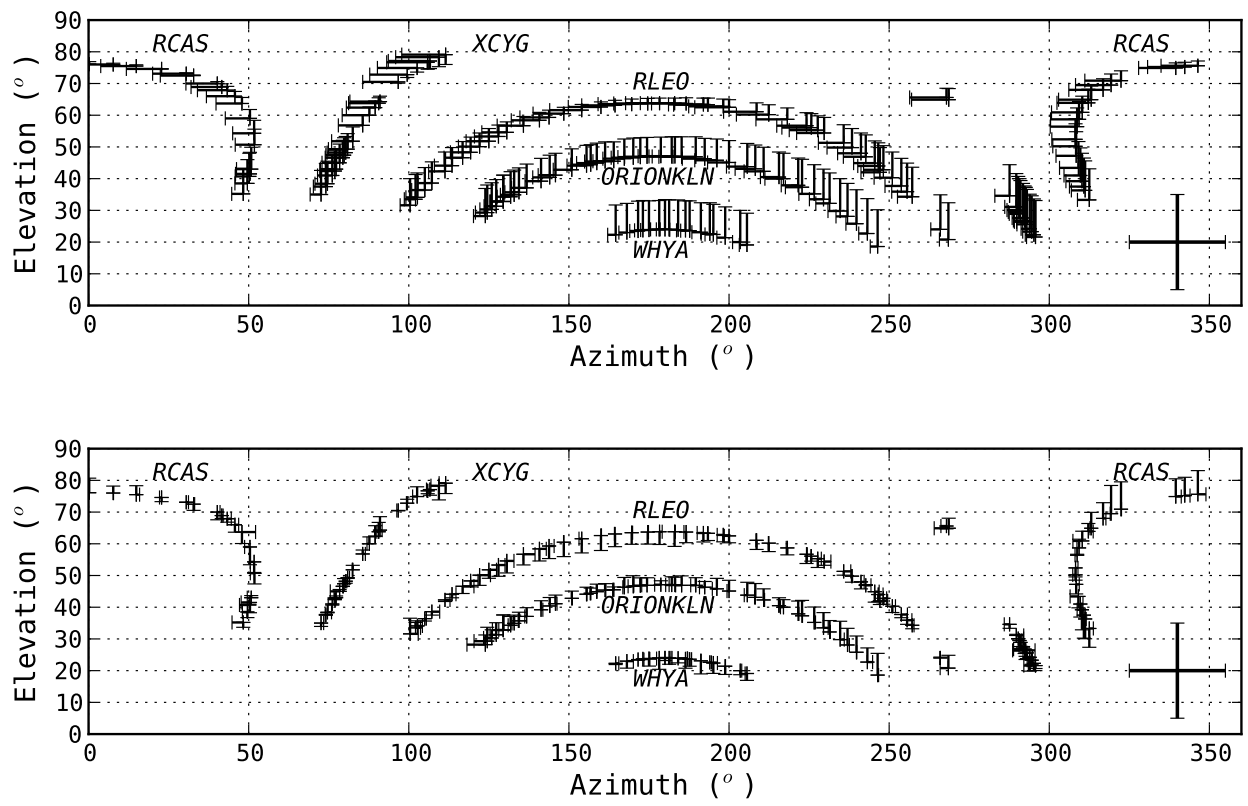

Fig. 3.- (Upper panel) Measured initial offsets in azimuth and elevation at the KVN Yonsei observatory. The size of the cross in the right bottom corner shows $300^{\prime \prime}$ in angular extent. (Lower panel) Measured residual offsets after the pointing model was applied. The size of the cross indicates $30^{\prime \prime}$, which is the estimated FWHM of the telescope. The pointing accuracy was $3.6^{\prime \prime} \mathrm{rms}$.

Table 2.

$\mathrm{SiO}$ maser sources observed

\begin{tabular}{lcc}
\hline \hline & $\begin{array}{c}\alpha(\mathrm{J} 2000) \\
\text { Source }\end{array}$ & $\begin{array}{c}\left.\text { ( } \begin{array}{c}\mathrm{m} \\
\mathrm{m}\end{array}\right) \\
\left({ }^{\circ}{ }^{\prime \prime}{ }^{\prime \prime}\right)\end{array}$ \\
\hline IK Tau $^{\mathrm{a}}$ & $03: 53: 28.84$ & $+11: 24: 22.6$ \\
TX Cam $^{\mathrm{a}}$ & $05: 00: 50.39$ & $+56: 10: 52.6$ \\
Orion KL & $05: 35: 14.51$ & $-05: 22: 30.6$ \\
R Leo $^{\mathrm{b}}$ & $09: 47: 33.49$ & $+11: 25: 43.6$ \\
W Hya & $13: 49: 02.00$ & $-28: 22: 03.5$ \\
$\chi$ Cyg $^{\mathrm{b}}$ & $19: 50: 33.92$ & $+32: 54: 50.6$ \\
R Cas & $23: 58: 24.87$ & $+51: 23: 19.7$ \\
\hline
\end{tabular}

a Observed only with the KVN Tamna telescope.

b Observed with the KVN Yonsei and Ulsan telescopes.

$\mathrm{SiO}(v=0, \mathrm{~J}=2-1)$ line. It has 256 channels with $64-$ $\mathrm{MHz}$ bandwidth, which gives a velocity resolution of $0.87 \mathrm{k} \mathrm{ms}^{-1}$.

A powermeter (Agilent E4417A EPM-P Series) was used for the measurements of various efficiencies by observing planets and the Moon in the $97-\mathrm{GHz}$ continuum emission. The typical linearity error is $<2 \%$ at normal output power, which is about $-20 \mathrm{dBm}$.

\section{CONTROL SOFTWARE}

Even though KVN will be usually operated as a VLBI network, software for single-dish observation is essential. KVN single-dish observation software supports basic observing modes such as sky dipping, position switching, focusing, five-point mapping, and onthe-fly (OTF) mapping. Most functions are written in Python language. This control software runs on a PC running Linux.

During spectroscopic observations, the data taking process also performs baseline subtraction, five-point fitting, and display of the observed data, and then writes the data in Gildas CLASS file format. Continuum data taken with the powermeter at 100 millisecond intervals are written out in ASCII text format together with header files, which contain antenna position and calibration information. The continuum OTF raw data are re-sampled to regular-grid images and the reduced images are displayed by SAOImage DS9 incorporated with a Python module. For operational convenience, the graphical user interface is developed using wxPython module.

The antenna control software runs on a Power PC running real-time operating system VxWorks, which is guaranteed to execute tasks without latency at accurate periods. The desired velocities of both azimuth and elevation axes are updated every 4 milliseconds by the antenna control software based on the encoder readout and velocities. The measured tracking accuracy is $<0.5^{\prime \prime} \mathrm{rms}$. 


\section{MEASUREMENTS OF POINTING AC- CURACY}

We measured the pointing accuracies of the KVN 21$m$ telescopes by observing five $\mathrm{SiO}$ maser sources in the $\mathrm{SiO}(v=1, \mathrm{~J}=2-1)$ line at $86.24343 \mathrm{GHz}$ (Table 2$)$. All the spectra were obtained on the $T_{A}^{*}$ scale by the standard chopper-wheel method (Kutner \& Ulich 1981). The sources were carefully selected such that their trajectories roughly covered the entire sky. While tracking each source, we made five-point mappings repeatedly and measured the offsets of azimuth and elevation. The upper panel in Figure 3 shows the offsets on the azimuth-elevation space for the KVN Yonsei telescope. A pointing model was built based on the measured offsets. In the model we take into account azimuth bearing warping and empirically correct for atmospheric refraction in addition to misalignments of the antenna axes and gravitational deformation (Mangum 2001). After applying the pointing model, the offset measurement procedure was repeated. Fig. 3 (lower panel) displays the residual offsets and Table 3 presents the rms of them for each telescope. The values are $3.6^{\prime \prime}$, $5.3^{\prime \prime}, 3.6^{\prime \prime}$ for Yonsei, Ulsan, and Tamna sites, respectively.

After constructing the pointing model, we conducted acceptance tests on the KVN 21-m antennae following the procedure agreed with Antedo. The procedure is summarized as follows.

1. By five-point mapping, measure the pointing offset on a source and correct it.

2. For two hours, repeat the five-point mapping and record the pointing offsets without any correction as long as the source is within 10 degrees from the starting position.

3. Repeat the steps above while tracking the source.

We performed tests on four of the five $\mathrm{SiO}$ maser sources observed in constructing the pointing model at each site, under the condition that the wind speed $<10 \mathrm{~m} \mathrm{~s}^{-1}$.

Table 4 exhibits the results. While nearly all data sets have $<4^{\prime \prime} r m s$ at the KVN Yonsei and Ulsan sites, only $31 \%$ of the 26 data sets of the KVN Tamna site have $<4^{\prime \prime} \mathrm{rms}$ during the first observing run of 2008 September. However, we obtained approximately twice higher passed rate, $67 \%$ (18/27), during the second observing run in 2008 December. Since no significant change was made on the telescope system, we concluded that the low passed rate of the first observing run might be due to the atmosphere, especially anomalous refraction, rather than the telescope system. There have been some reports from other observatories that anomalous atmospheric refraction can cause large (few tens of arcseconds) random pointing errors of the telescope on a time scale of a few minutes in the 100$\mathrm{GHz}$ band (e.g., Altenhoff et al. 1987). The details of this issue will be addressed in another paper (Kim et al., in preparation). There were still a couple of outliers

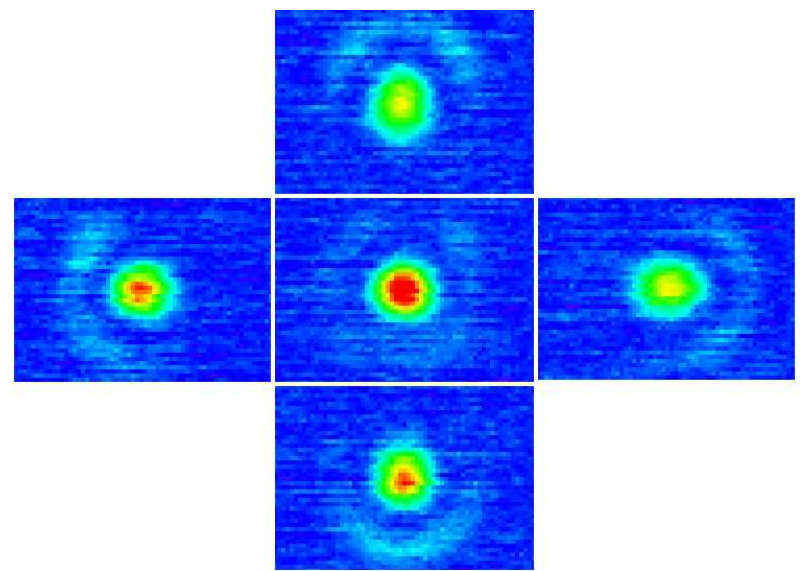

Fig. 4. - The OTF image samples of Saturn made during the subreflector adjustment at the KVN Ulsan radio telescope. The peak intensity of the planet and the morphology and level of the first sidelobe vary with the position and angle of the subreflector with respect to the primary reflector and the feed horn. The central image shows the highest intensity of Saturn at a given elevation together with the lowest level of the first sidelobe, which is approximately circularly symmetric. Each image is $3.1^{\prime} \times 2.3^{\prime}$ in size.

Table 3.

Pointing accuracy measurements

\begin{tabular}{|c|c|c|c|}
\hline Telescope & $\begin{array}{c}\text { Azimuth } \\
\left({ }^{\prime \prime}\right)\end{array}$ & $\begin{array}{c}\text { Elevation } \\
\left({ }^{\prime \prime}\right)\end{array}$ & $\begin{array}{c}\theta \\
\left({ }^{\prime \prime}\right)\end{array}$ \\
\hline Yonsei & 1.65 & 3.16 & 3.6 \\
\hline Ulsan & 2.28 & 4.83 & 5.3 \\
\hline Tamna & 1.98 & 2.95 & 3.6 \\
\hline
\end{tabular}

in each of the remaining 9 data sets. These were also likely due to the anomalous atmospheric refraction. If the outliers are excluded in each set, the passed rate would be $89 \%(24 / 26)$. This is comparable to the rates of the other two sites.

\section{MEASUREMENTS OF EFFICIENCIES}

We mapped the planets in the $97-\mathrm{GHz}$ continuum emission by the OTF mapping technique for measuring the main beam size and various efficiencies. All the continuum data were strictly calibrated by the hot-cold load method in which the ambient and liquid nitrogen temperature absorbers were put alternatively in front of the receiver feed horn (Kutner \& Ulich 1981).

Before the efficiency measurements we adjusted the subreflector's position with respect to the main reflector using the hexapod. This procedure was required to compensate the sagging of the subreflector itself as well 
Table 4.

Acceptance test for pointing accuracy

\begin{tabular}{|c|c|c|c|c|c|c|c|c|c|}
\hline \multirow[b]{2}{*}{ Source } & \multicolumn{2}{|c|}{ Yonsei } & \multicolumn{2}{|c|}{ Ulsan } & \multicolumn{2}{|c|}{ Tamna I } & \multicolumn{3}{|c|}{ Tamna II ${ }^{\mathrm{a}}$} \\
\hline & $\mathrm{N}_{1}{ }^{\mathrm{b}}$ & $\mathrm{N}_{2}{ }^{\mathrm{c}}$ & $\mathrm{N}_{1}$ & $\mathrm{~N}_{2}$ & $\mathrm{~N}_{1}$ & $\mathrm{~N}_{2}$ & $\mathrm{~N}_{1}$ & $\mathrm{~N}_{2}$ & $\mathrm{~N}_{3}{ }^{\mathrm{d}}$ \\
\hline IK Tau & $\cdots$ & $\ldots$ & $\ldots$ & $\ldots$ & 11 & 4 & $\ldots$ & $\ldots$ & $\ldots$ \\
\hline TX Cam & $\ldots$ & $\ldots$ & $\ldots$ & $\ldots$ & 5 & 1 & $\ldots$ & $\ldots$ & $\ldots$ \\
\hline Orion KL & 6 & 6 & 8 & 8 & 4 & 3 & 9 & 8 & 9 \\
\hline R Leo & 8 & 8 & 10 & 10 & 6 & 0 & 9 & 7 & 8 \\
\hline$\chi \mathrm{Cyg}$ & 7 & 7 & 8 & 7 & $\ldots$ & $\ldots$ & $\ldots$ & $\ldots$ & $\ldots$ \\
\hline R Cas & 6 & 4 & 8 & 7 & $\ldots$ & $\cdots$ & 9 & 2 & 7 \\
\hline In total & 27 & $\begin{array}{c}25 \\
(93 \%)\end{array}$ & 34 & $\begin{array}{c}32 \\
(94 \%)\end{array}$ & 26 & $\begin{array}{c}8 \\
(31 \%)\end{array}$ & 27 & $\begin{array}{c}18 \\
(67 \%)\end{array}$ & $\begin{array}{c}24 \\
(89 \%)\end{array}$ \\
\hline
\end{tabular}

aTamna I made in 2008 September while Tamna II made in 2008 December.

b The number of data sets obtained.

"The number of data sets with pointing accuracies $<4^{\prime \prime} \mathrm{rms}$.

d The number of data sets with pointing accuracies $<4^{\prime \prime} \mathrm{rms}$ after a couple of outliers are excluded.

as the deformation of the primary reflector by gravity with the elevation. We created a model of the subreflector adjustment for each telescope by determining the best sets of the positional parameters, (X, Y, Z, Tip, Tilt), at two very different elevations. Z, X, and $\mathrm{Y}$ are respectively, the displacements along the shaped Cassegrain axis, along the axis parallel to the elevation axis and along the axis perpendicular to the previous two. The Tip and Tilt parameters are rotations around the $\mathrm{X}$ - and the $\mathrm{Y}$-axis respectively. Here we mean the best set of parameters by which the highest main peak intensity are obtained at a given elevation, together with the lowest level and circularly symmetric morphology of the first sidelobe. Fig. 4 shows sample images obtained by adjusting the subreflector.

We first determined the FWHM of the main beam from the continuum images of the planets. The estimated beam sizes of all three telescopes are about $30^{\prime \prime}$, which is equivalent to the diffraction limit of the $21-\mathrm{m}$ telescope at the observing frequency. This implies that the illumination of the main reflector is almost uniform, as expected. With uniform illumination, the first sidelobe is expected to be $\sim 1.6$ times FWHM away from the main beam center and the level be $-17.6 \mathrm{~dB}$ (Rohlfs \& Wilson 1996). For all three telescopes, we estimated the first sidelobe levels to be $-14 \pm 1 \mathrm{~dB}$, which is quite higher than the theoretical value (Fig. 5). They actually appear about $50^{\prime \prime}(\sim 1.6 \times \mathrm{FWHM})$ from the main beam centers. For comparison, the first sidelobe levels are usually between $-30 \mathrm{~dB}$ and $-20 \mathrm{~dB}$ for the conventional Cassegrain antennae.

We measured the aperture efficiency, $\eta_{\mathrm{A}}$, and mainbeam efficiency, $\eta_{\mathrm{mb}}$, from the OTF images of Venus using the following equations (Schloerb \& Snell 1980; Rho \& Jung 1999; Koo et al. 2003).

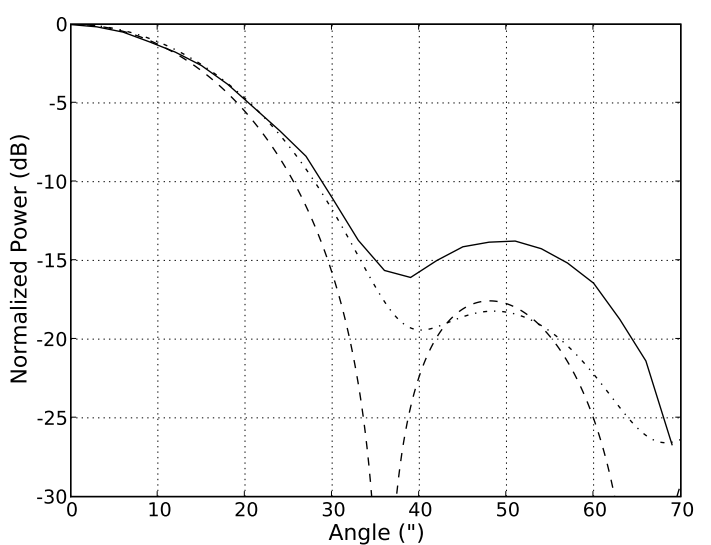

Fig. 5. - The solid line shows the normalized radial intensity profile of Venus observed by the KVN Yonsei telescope on 2009 January 5 . The intensity was azimuthally averaged. Venus was $22^{\prime \prime}$ in size. The first sidelobe appears around $50^{\prime \prime}$ from the main peak and the level is about $-14 \mathrm{~dB}$. The dashed line displays the beam pattern of a 21-m telescope with the perfect uniform illumination, while the dotted line exhibits the expected radial intensity profile of a source with the same size as Venus, as observed with the hypothetical telescope. The first sidelobe appears at a similar position but the level is twice lower.

$$
\begin{gathered}
\eta_{\mathrm{A}} \equiv \frac{A_{\mathrm{e}}}{A_{\mathrm{p}}}=\frac{\lambda^{2} T_{\mathrm{A}}^{*}}{A_{\mathrm{p}} T_{\mathrm{b}} \Omega_{\mathrm{s}}}, \\
\eta_{\mathrm{mb}} \equiv \frac{\Omega_{\mathrm{M}}}{\Omega_{\mathrm{A}}}=\frac{T_{\mathrm{A}}^{*} \Omega_{\mathrm{M}}}{T_{\mathrm{b}} \Omega_{\mathrm{s}}}=\frac{A_{\mathrm{p}} \Omega_{\mathrm{M}}}{\lambda^{2}} \eta_{\mathrm{A}}, \\
\Omega_{\mathrm{s}}=\Omega_{\mathrm{M}}\left[1-\exp \left(-\ln 2\left(\theta_{\mathrm{s}} / \theta_{\mathrm{M}}\right)^{2}\right)\right],
\end{gathered}
$$


Table 5.

Aperture and main beam efficiency measurements

\begin{tabular}{lcccccc}
\hline \hline Telescope & $\begin{array}{c}\text { Observing } \\
\text { Date }\end{array}$ & $\begin{array}{c}\text { Zenith } \\
\text { Opacity }\end{array}$ & $\begin{array}{c}\text { Venus Diameter } \\
\left({ }^{\prime \prime}\right)\end{array}$ & $\begin{array}{c}\text { Elevation } \\
\left({ }^{\circ}\right)\end{array}$ & $\begin{array}{c}\eta_{\mathrm{A}} \\
(\%)\end{array}$ & $\begin{array}{c}\eta_{\mathrm{mb}} \\
(\%)\end{array}$ \\
\hline Yonsei & 2009 Jan 05 & 0.09 & 22 & 40 & 52 & 46 \\
Ulsan & 2009 Apr 23 & 0.13 & 45 & 57 & 53 & 47 \\
Tamna & 2008 Dec 09 & 0.12 & 18 & 35 & 51 & 45 \\
\hline
\end{tabular}

$$
\Omega_{\mathrm{M}}=1.133 \theta_{\mathrm{M}}^{2}
$$

Here $\lambda, T_{\mathrm{b}}, \theta_{\mathrm{s}}, \theta_{\mathrm{M}}$ are the observing wavelength, the brightness temperature, the angular size of the source, and the size of the main beam (FWHM), respectively. We assumed that (1) the main beam is Gaussian in shape, (2) Venus is a disk with uniform brightness, and (3) the brightness temperature is $367 \pm 10 \mathrm{~K}$ (Ulich 1981). The estimated aperture efficiencies are about $52 \%$ at all the three telescopes. This value is somewhat lower than $60 \%$ mentioned in the proposal of Antedo, but is similar to $56 \%$ calculated by Hay (2008). Both calculations assumed that the surface accuracy of the main reflector is $\sim 150 \mu \mathrm{m}$ and that there is no misalignment in the antenna optics. Hay (2008) estimated the efficiency by applying a physical optics analysis, which accounts for diffraction effects, with a slightly lower blockage efficiency than used by Antedo, $88 \%$ vs. $93 \%$, by considering the cladding of the subreflector supports. The main beam efficiencies are about $46 \%$. Table 5 summarizes the observational details and the results.

The first assumption may cause uncertainty in the efficiency estimation because the main beam is actually more like a Bessel function due to the uniform illumination. According to our calculations, the error depends on the size ratio of the source to the main beam. The derived efficiency is overestimated by $<0.5 \%$ for the ratio $\leq 1.0$ (Yonsei and Tamna), whereas it is underestimated by about $1 \%$ for the ratio $\simeq 1.5$ (Ulsan). The third assumption can produce larger uncertainty. The measurement error is equivalent to $\pm 1.4 \%$. Moreover, the measurement was made at $90 \mathrm{GHz}$. It has been found from observational and theoretical studies that the brightness temperature of Venus gradually decreases with frequency and it ranges between $350 \mathrm{~K}$ and $360 \mathrm{~K}$ at $97 \mathrm{GHz}$ depending on the atmospheric models (Ulich 1981; Fahd et al. 1992). If that is the case, the efficiency is underestimated by $1.0 \%-2.4 \%$.

We derived the moon efficiency, $\eta_{\text {moon }}$, of the KVN Tamna radio telescope by the mapping the Moon at the same frequency, following the procedure presented by Mangum (1993) (see Linsky 1973 for more details). The moon efficiency is defined as the ratio of the antenna temperature of the Moon to the brightness tem- perature. It can be practically used as the forward spillover and scattering efficiency, $\eta_{\mathrm{fss}}$ (Kutner \& Ulich 1981). The brightness temperature of the Moon is calculated using the equation below.

$$
\begin{aligned}
T_{\mathrm{b}, \text { moon }}(\lambda)= & T_{0}(0)+\frac{0.77 T_{0}(0)}{\left[1+0.48 \lambda+0.11 \lambda^{2}\right]^{1 / 2}} \\
& \times \cos \left[\chi-\tan ^{-1}\left(\frac{0.24 \lambda}{1+0.24 \lambda}\right)\right]
\end{aligned}
$$

where

$$
\chi=\frac{\phi}{P} \times 360-180
$$

Here $\phi$ is the days since new Moon, $P$ is the period of the Moon, 29.530589 days, and $T_{0}(0)$ is $227.7 \pm 8.9$ around $100 \mathrm{GHz}$. The estimated efficiency is about $84 \%$ (Table 6). This implies that the contribution of the sidelobes is comparable to that of the main beam, taking into account that the main beam efficiency is $45 \%$ for the telescope. In case of the classical Cassegrain telescopes the contribution of the sidelobes is much lower. For example, the moon and main beam efficiencies of IRAM $30 \mathrm{~m}$ are $94 \%$ and $73 \%$, respectively, around $90 \mathrm{GHz}$ (Greve et al. 1998). Such a large contribution of the sidelobes might stems from the aforementioned fact that KVN telescopes have much higher sidelobe levels than the Cassegrain ones. Since the other two have almost the same main beam size, aperture and main beam efficiencies as the KVN Tamna telescope, one can expect similar moon efficiencies for them.

\section{SUMMARY}

The construction of the KVN 21-m radio telescopes was completed in 2008 December. We determined the parameters of the telescopes by observing astronomical objects, such as $\mathrm{SiO}$ maser sources, planets, and the Moon. The measured pointing accuracies are about $4^{\prime \prime} \mathrm{rms}$ for all three telescopes. The beam sizes are about $30^{\prime \prime}$, which is nearly the diffraction limit of the 21-m telescope at the observing frequency $(97 \mathrm{GHz})$. The aperture and main-beam efficiencies were derived to be about $52 \%$ and $46 \%$, respectively. The moon 
Table 6.

Moon efficiency measurements

\begin{tabular}{lcccccc}
\hline \hline Telescope & $\begin{array}{c}\text { Observing } \\
\text { Date }\end{array}$ & $\begin{array}{c}\mathrm{UT} \\
(\mathrm{h}: \mathrm{m})\end{array}$ & $\begin{array}{c}\phi \\
(\text { Days })\end{array}$ & $\begin{array}{c}T_{\mathrm{b}} \\
(\mathrm{K})\end{array}$ & $\begin{array}{c}T_{\mathrm{A}}^{*} \\
(\mathrm{~K})\end{array}$ & $\begin{array}{c}\eta_{\text {moon }} \\
(\%)\end{array}$ \\
\hline Tamna & 2008 Sep 18 & $14: 10$ & 18.8 & 311.3 & 262.5 & 84 \\
\hline
\end{tabular}

efficiency was estimated to be $\sim 84 \%$ for the $\mathrm{KVN}$ Tamna telescope. The first sidelobes appear around $50^{\prime \prime}$ from the main beam centers and the levels are about $-14 \mathrm{~dB}$, which is significantly higher than the typical level $(<-20 \mathrm{~dB})$ of the classical Cassegrain antennae.

\section{ACKNOWLEDGMENTS}

We are very grateful to many observers for performing parts of the observations. They are as follows: Jaesang Cho, Eun Jung Chung, Yukitoshi Kan-Ya, Jiman Kang, Chang Hee Kim, Gwanjeong Kim, Jaeheon Kim, Mi Kyung Kim, Jeewon Lee, Jeong Ae Lee, Sang-Sung Lee, Chung Sik Oh, Junghwan Oh, Duk-Gyoo Rho, and Hahn Yi. We also thank the anonymous referee for helpful comments.

\section{REFERENCES}

Altenhoff, W. J., Baars, J. W. M., Wink, J. E., \& Downes, D. 1987, Observations of Anomalous Refraction at Radio Wavelengths, A\&A, 184, 381

Asaki, Y., Shibata, K. M., Kawabe, R., Roh, D.-G., Saito, M., Morita, K.-I., \& Sasao, T. 1998, Phase Compensation Experiments with the Paired Antennas Method 2. Millimeter-wave Fringe Correction Using Centimeter-Wave Reference, Radio Science, 33,1297

Chung, M.-H., Byun, D.-Y., \& Khaikin, V. B. 2006, Gain Degradation of KVN 21-m Shaped Cassegrain Antenna due to Misalignment of Antenna Optics, Journal of Astronomy and Space Sciences, 23, 327

Fahd, A. K., et al. 1992, NASA Technical Report No. 1992-1: Study and Interpretation of the Millimeter Wave Spectrum of Venus, 105

Green, K. A. 1963, Modified Cassegrain Antenna for Arbitrary Aperture Illumination, IEEE Transactions on Antennas and Propagation, 11, 589

Greve, A., Kramer, C., \& Wild, W. 1998, The Beam Pattern of the IRAM 30-m Telescope, A\&AS, 133, 271

Han, S.-T., Lee, J.-W., Kang, J., Je, D.-H., Chung, M.-H., Wi, S.-O., Sasao, T., \& Wylde, R. 2008, Millimeter-Wave Receiver Optics for Korean VLBI Network, International Journal of Infrared and Millimeter Waves, 29, 69
Hay, S. 2008, Calculated KVN 21-m Antenna Efficiency

Jung, T., Sohn, B. W., Kobayashi, H., Sasao, T., Hirota, T., Kameya, O., Choi, Y. K., \& Chung, H. S. 2011, First Simultaneous Dual-Frequency Phase Referencing VLBI Observation with VERA, PASJ, 63,375

Kim, H.-G., Han, S.-T., Sohn, B. W., Oh, S.-J., Je, D.-H., Wi, S.-O., \& Song, M.-G. 2004, Construction of the Korean VLBI Network (KVN), Proceedings of the 7th Symposium of the European VLBI Network on New Developments in VLBI Science and Technology, 281

Koo, B,-C., Park, Y.-S., Hong, S. S., Yun, H.-S., Lee, S.-G., Byun, D.-Y., Lee, J.-W., Choi, H.-K., Lee, S.-S., Yoon, Y.-Z., Kim, K.-T., Kang, H. W., Lee, J.-E. 2003, Performance of the SRAO 6-Meter Radio Telescope, JKAS, 36, 43

Kutner, M. L., \& Ulich, B. L. 1981, Recommendations for Calibration of Millimeter-Wavelength Spectral Line Data, ApJ, 250, 341

Linsky, J. L. 1973, The Moon as a Proposed Radiometric Standard for Microwave and Infrared Observations of Extended Sources, ApJS, 25, 163

Mangum, J. G. 1993, Main-Beam Efficiency Measurements of the Caltech Submillimeter Observatory, PASP, 105, 117

Mangum, J. G. 2001, ALMA Memo 366: A Telescope Pointing Algorithm for ALMA

Middelberg, E., Roy, A. L., Walker, R. C., \& Falcke, H. 2005, VLBI Observations of Weak Sources Using Fast Frequency Switching, A\&A, 433, 897

Rho, D.-G., \& Jung, J. H. 1999, Characteristics of TRAO 14-m Radio Telescope, 14, 123

Rohlfs, K., \& Wilson, T. L. 1996, Tools of Radio Astronomy (Berlin: Springer-Verlag), 137-141

Schloerb, F. P., \& Snell, R. L. 1980, FCRAO Report No. 150

Ulich, B. L. 1981, Millimeter-Wavelength Continuum Calibration Sources, AJ, 86, 1619

Yi, J., \& Jung, T.-H. 2008, Image Simulations for the KVN Using the VLBA Image of SiO Masers, PKAS, 23,37 